\title{
Prevalence of Merkel cell polyomavirus in Merkel cell carcinoma
}

\author{
Eric J Duncavage, Barbara A Zehnbauer and John D Pfeifer \\ Lauren V. Ackerman Laboratory of Surgical Pathology, Division of Anatomic and Molecular Pathology, \\ Department of Pathology and Immunology, Washington University Medical Center, St Louis, MO, USA
}

\begin{abstract}
It has recently been shown that Merkel cell carcinoma, a rare and often lethal cutaneous malignancy, frequently harbors a novel clonally integrated polyomavirus aptly named Merkel cell polyomavirus. We aimed to study the prevalence of Merkel cell polyomavirus in cases of Merkel cell carcinoma, using specimens from formalin-fixed, paraffin-embedded tissue blocks. In our archives we identified 41 cases of Merkel cell carcinoma (from 29 different patients). Of these, 20 cases were primary cutaneous tumors, 4 were local recurrences, and 17 were metastases. PCR using two previously published primer sets, LT1 (440 bp amplicon) and LT3 (308 bp amplicon), as well as a novel primer set MCVPS1 (109 bp amplicon), was performed on all cases. Selected PCR products were sequenced to confirm amplicon identity. In addition, the MCVPS1 products were digested with BamH1, yielding an $83 \mathrm{bp}$ product. Amplifiable DNA was recovered in all 41 study cases. The detection rate of Merkel cell polyomavirus for each of the three primer sets was 22 of 29 patients (76\%) for MCVPS1, 12 of 29 (41\%) for LT3, and 8 of $29(28 \%)$ for LT1. The variation between primer set detection rates was largely due to poor DNA quality, as supported by poor amplification of the higher molecular weight markers in size control ladder products and the fact that all cases that were positive by LT1 and LT3 were positive by MCVPS1. Our findings provide further evidence to link Merkel cell polyomavirus with a possible role in the oncogenesis of Merkel cell carcinoma. On a more practical level, our paraffin-optimized primer set may be used as an ancillary test to confirm the diagnosis of Merkel cell carcinoma in the clinical setting or for screening other rare tumor types for the causative virus, especially those tumor types that are underrepresented in frozen tissue repositories.

Modern Pathology (2009) 22, 516-521; doi:10.1038/modpathol.2009.3; published online 27 February 2009
\end{abstract}

Keywords: Merkel cell carcinoma; polyomavirus; viral oncogenesis; Merkel cell polyomavirus

Merkel cell carcinoma is a rare and often lethal cutaneous malignancy that was originally described as 'trabecular carcinoma of the skin' in $1972 .{ }^{1}$ Although the cell of origin was traditionally thought to be the slow-acting mechanoreceptor Merkel cell located in the epidermis, ${ }^{2}$ recent studies have cast doubt on this assumption. ${ }^{3,4}$ Histologically, Merkel cell carcinoma is composed of small- to intermediatesized round blue cells that have an oval nucleus and scant cytoplasm. The cells are typically arranged in sheets or nests within the dermis, often extending into the subcutis (Figure 1). Immunohistochemically, Merkel cell carcinoma typically shows reactivity to both epithelial and neuroendocrine markers, including pan-cytokeratin, epithelial membrane antigen, chromogranin, and synaptophysin. Immunostains for

Correspondence: Dr EJ Duncavage, Department of Pathology, Washington University School of Medicine, Box 8118, 660 Euclid Ave, St Louis, MO 63110-1093, USA.

E-mail: eduncavage@path.wustl.edu

Received 7 October 2008; revised and accepted 7 January 2009; published online 27 February 2009 cytokeratin 20 (CK20) usually show a characteristic 'dot-like' perinuclear staining pattern. ${ }^{5}$

Merkel cell carcinoma is most common among older ( $>80$ years) white men and is largely absent in populations less than 40 years old. Although the estimated annual incidence of Merkel cell carcinoma in the United States is only about 470 cases a year, there has been a threefold increase of cases since $1986 .{ }^{6}$ Interestingly, the incidence of Merkel cell carcinoma is significantly higher in populations with organ transplants, lymphoma, and reduced immunity; $;^{7,8}$ patients with HIV have a relative risk for the tumor of 13.4 compared with the general population. ${ }^{9}$

Merkel cell carcinoma was recently shown to harbor a novel polyomavirus aptly named the Merkel cell polyomavirus, in the majority of cases. ${ }^{10,11}$ The virus is a nonenveloped, doublestranded DNA virus with a $5387 \mathrm{bp}$ circular genome that shares a high degree of homology with African green monkey polyomavirus viral proteins and T-cell antigens. In common with other polyomaviruses that infect humans (including BK virus, JC 
a
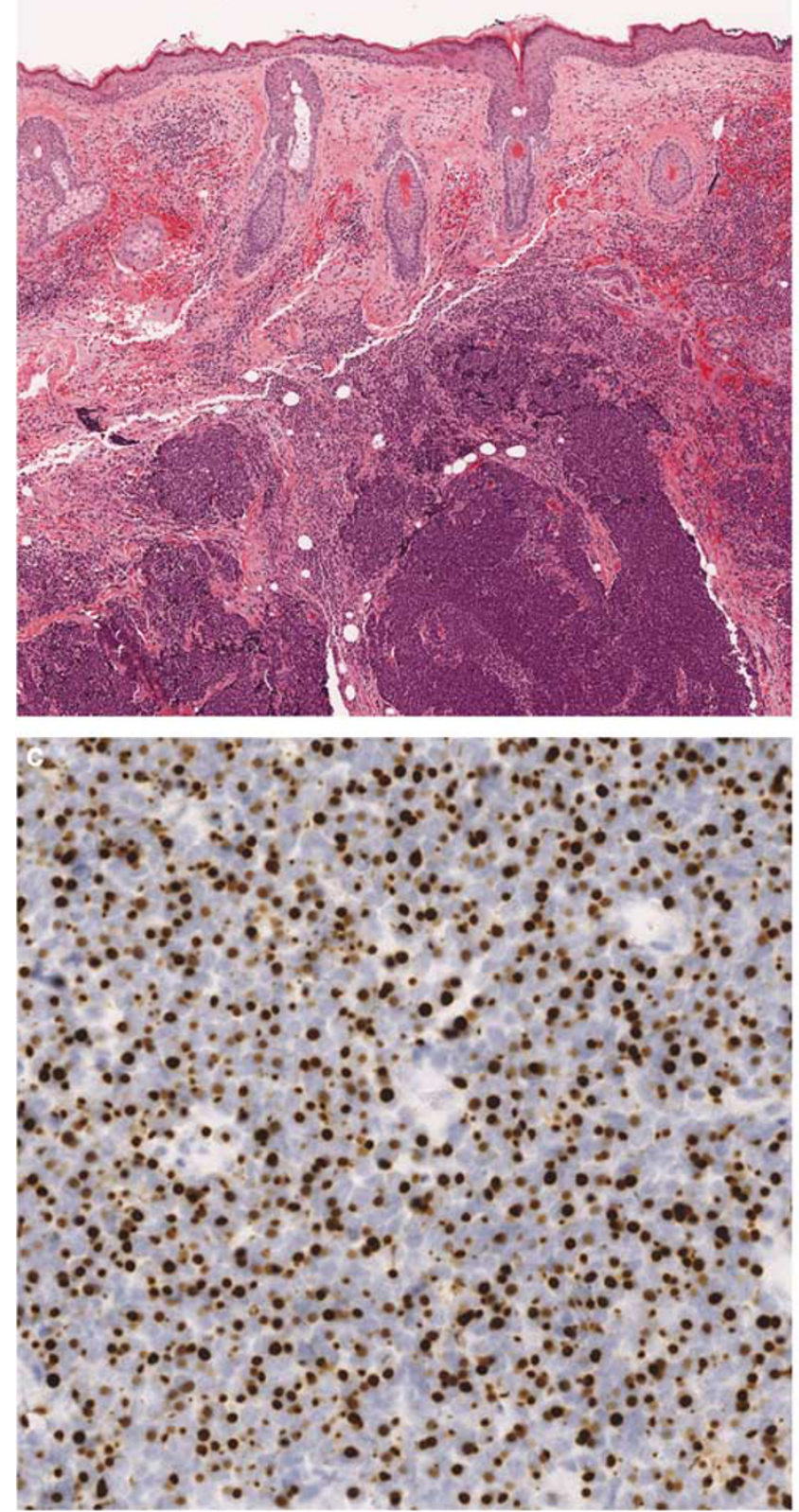
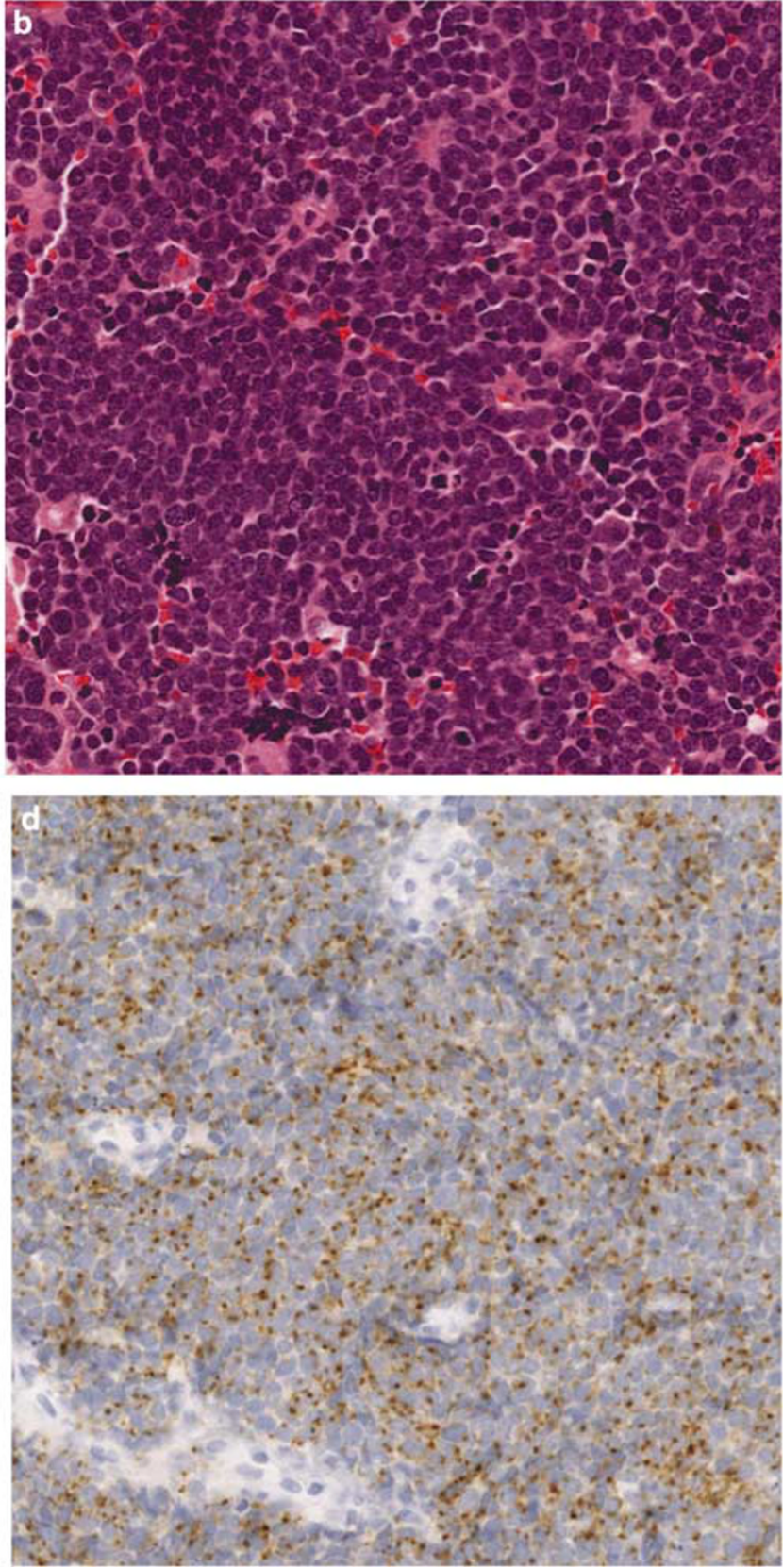

Figure 1 Histological features of Merkel cell carcinoma. (a) Low-power view of a primary Merkel cell carcinoma occurring in the head and neck region. The nested neoplastic cell population is confined to the deep dermis and extends into subcutaneous fat (original magnification, $\times 4$; H\&E stain). (b) High-power view showing a monotonous population of neoplastic cells with large nuclei, fine chromatin, and inconspicuous nucleoli. Mitotic figures are rare in this specimen (original magnification, $\times 20$; H\&E stain). (c) CK20 immunostaining shows characteristic punctuate perinuclear staining (original magnification, $\times 20$ ). (d) Chromogranin immunostaining shows a neuroendocrine pattern (original magnification, $\times 20$ ).

virus, KI virus, and WU virus), Merkel cell polyomavirus expresses a large T-cell antigen. However, unlike the other human polyomaviruses that have never been associated with neoplastic transformation of any cell type, Merkel cell polyomavirus has been implicated in oncogenesis (primarily based on the demonstration of clonal insertion of the virus in cases of Merkel cell carcinoma). ${ }^{10}$ Although the direct association of Merkel cell polyomavirus with a particular tumor type is unique among human polyomaviruses, it is important to note that other nonhuman polyomaviruses, including SV40, have long been associated with sarcomas in other mammals. ${ }^{12,13}$

As the incidence of Merkel cell carcinoma is so low, and prospectively collected frozen tissue specimens are rare, an accurate assessment of the prevalence of Merkel cell polyomavirus in cases of Merkel cell carcinoma is most readily performed by analysis of formalin-fixed, paraffin-embedded 
tissue. Our study was designed to perform such an analysis.

\section{Materials and methods}

\section{Case Selection}

A search of the files of the Lauren $\mathrm{V}$ Ackerman Laboratory of Surgical Pathology at Washington University Medical Center from January 1989 to January 2008 identified 41 cases of surgically resected Merkel cell carcinoma, including both cutaneous malignancies and metastases, for which formalin-fixed, paraffin-embedded tissue blocks were available. Of the 41 cases, 29 were from unique patients; 20 cases were from primary cutaneous excisions, 17 from metastases, and 4 from recurrences (Table 1). All cases were reviewed by a pathologist to confirm the diagnosis. Blocks and slides were aligned and representative areas were cored with $2 \mathrm{~mm}$ sterile punches (Miltex, Tuttlingen, Germany).

\section{PCR}

The $2 \mathrm{~mm}$ tumor cores were deparaffinized and subjected to $48 \mathrm{~h}$ proteinase- $\mathrm{K}$ digestion at $55^{\circ} \mathrm{C}$. DNA was then extracted using the Puregene Extraction kit (Gentra Scientific, Minneapolis, MN, USA) per the manufacturer's instructions. The resulting DNA was assayed for concentration and protein contamination to make it possible to standardize template additions to the PCR amplifications. PCR was performed using the Platinum Taq HF kit (Invitrogen, Carlsbad, CA, USA) with approximately $100 \mathrm{ng}$ of genomic DNA for each sample. Primers for $\beta$-globin and a multiplexed primer set for a size ladder (consisting of 100, 200, 300, 400, and $600 \mathrm{bp}$ products) ${ }^{14}$ were used as amplification controls. PCR amplifications consisted of 35 cycles of template denaturation at $94^{\circ} \mathrm{C}$ for $30 \mathrm{~s}$, annealing for $30 \mathrm{~s}$ at $50^{\circ} \mathrm{C}$ for LT1 and LT3, at $55^{\circ} \mathrm{C}$ for MCVPS1 and controls (details of the primer sets used to identify Merkel cell polyomavirus are shown in Table 2 and Figure 1), and extension at $68^{\circ} \mathrm{C}$ for $1 \mathrm{~min}$. The MCVPS1 primer set was designed in silico specifically for use with human formalin-fixed paraffinembedded tissue in that the primers have high melting temperatures, have low homology to human genomic DNA at other than the target sequences (the primers have no binding sites within 50-1000 bp of one another in human genomic DNA ${ }^{15}$ ), produce a small amplicon, and generate an amplicon with an internal BamH1 site at position 83 to aid in PCR product validation. In addition, BLAST homology searches (http://blast.ncbi.nlm.nih.gov/Blast.cgi) comparing the MCVPS1 amplicon to all 855 known polyomaviruses in GenBank showed no significant homology, reducing the likelihood of cross reactivity with other polyomaviruses.

\section{PCR Product Verification}

Representative PCR products were cloned into vector pCR2.1 using the TA Cloning kit (Invitrogen), then sequenced using the Taq dideoxy terminator cycle sequencing kit (Applied Biosystems Inc., Foster City, CA, USA) and a fluorescent DNA sequencer (373A; Applied Biosystems Inc.). The resulting sequences were aligned against the Merkel cell polyomavirus sequence (NCBI gene accession number 165973999) and showed $100 \%$ homology to the predicted amplicon. For indirect product verification, the MCVPS1 products were digested with BamH1 (New England Biolabs, Ipswich, MA, USA) to yield a smaller $83 \mathrm{bp}$ product detected by agarose gel electrophoresis (Figure 2).

\section{Regulatory Approval}

This study was approved by the Human Studies Committee at Washington University School of Medicine.

\section{Results}

\section{Patient Population}

The average patient age at the time of resection was $71 \pm 12$ years (range $48-85$ years). Of the 29 unique cases, 20 were from men $(69 \%)$ and 9 (31\%) from women. The most common primary tumor site was the head/neck (10 of 17 cases with a documented primary site), followed by the extremities (4 cases), and the trunk ( 3 cases). Of 29 patients, 7 had a previous diagnosis of malignancy, including 3 cases of squamous-cell carcinoma, 2 cases of basal cell carcinoma, 1 case of colorectal adenocarcinoma, and 1 case of chronic lymphocytic lymphoma.

\section{Prevalence of Merkel Cell Polyomavirus}

The Merkel cell polyomavirus detection rate from formalin-fixed, paraffin-embedded tissue varied with the primer set used, ranging from 32 of 41 cases $(78 \%)$ for MCVPS1, to 9 of 41 cases (22\%) for LT1, and 17 of 41 cases (41\%) for LT3 (Table 1). Using the MCVPS1 primer set, we detected Merkel cell polyomavirus in 76\% (22 of 29) of unique cases. Cases that tested positive with the MCVPS1 primer set were subsequently confirmed by $\mathrm{BamH} 1$ digestion and the identification of an $83 \mathrm{bp}$ product (Figure 3).

The $110 \mathrm{bp} \beta$-globin product indicative of the presence of intact amplifiable DNA could be amplified from all 41 cases. However, both 100 and $200 \mathrm{bp}$ size control markers could be amplified from only 35 of 41 cases, and a $300 \mathrm{bp}$ marker from only 21 of 41 cases; 400 and $600 \mathrm{bp}$ markers could not be amplified from any cases. Given the decreased amplification efficiency of larger amplicons, a 
Table 1 Study cases

\begin{tabular}{|c|c|c|c|c|c|c|}
\hline Number & Case & Age & MCVPS1 & $L T 1$ & LT3 & Location \\
\hline 1 & Unique primary & 81 & neg & neg & neg & Head and neck \\
\hline 2 & Unique primary & 79 & + & neg & + & Head and neck \\
\hline 3 & Unique primary & 66 & neg & neg & neg & Head and neck \\
\hline 4 & Unique recurrence & 79 & + & neg & + & Head and neck \\
\hline 5 & Unique primary & 56 & + & neg & + & Extremity \\
\hline 6 & Duplicate case 2 & 79 & + & neg & + & \\
\hline 7 & Unique primary & 84 & neg & neg & neg & Extremity \\
\hline 8 & Unique primary & 72 & + & + & + & Trunk \\
\hline 9 & Duplicate case 3 & 66 & neg & neg & neg & \\
\hline 10 & Unique primary & 78 & + & neg & neg & Head and neck \\
\hline 11 & Unique primary & 87 & + & neg & neg & Head and neck \\
\hline 12 & Unique primary & 58 & + & neg & + & Trunk \\
\hline 13 & Unique primary & 75 & + & neg & + & Head and neck \\
\hline 14 & Unique primary & 69 & + & neg & neg & Head and neck \\
\hline 15 & Re-excision case 7 & 84 & + & neg & neg & \\
\hline 16 & Unique primary & 69 & + & neg & neg & Head and neck \\
\hline 17 & Unique primary & 70 & + & neg & + & Head and neck \\
\hline 18 & Unique primary & 83 & + & + & + & Head and neck \\
\hline 19 & Recurrence case 18 & 84 & neg & neg & neg & \\
\hline 20 & Metastasis case 10 & 78 & neg & neg & neg & \\
\hline 21 & Unique metastasis & 67 & neg & neg & neg & \\
\hline 22 & Metastasis case 13 & 75 & + & neg & neg & \\
\hline 23 & Metastasis case 12 & 86 & + & neg & + & \\
\hline 24 & Recurrence case 31 & 49 & + & + & + & \\
\hline 25 & Unique primary & 82 & + & neg & + & Extremity \\
\hline 26 & Unique metastasis & 70 & + & neg & neg & \\
\hline 27 & Metastasis case 25 & 82 & + & neg & neg & \\
\hline 28 & Unique metastasis & 69 & + & + & + & \\
\hline 29 & Unique metastasis & 70 & neg & neg & neg & \\
\hline 30 & Metastasis case 16 & 69 & + & neg & neg & \\
\hline 31 & Unique primary & 48 & + & + & + & Head and neck \\
\hline 32 & Unique primary & 56 & + & + & neg & Extremity \\
\hline 33 & Unique metastasis & 52 & + & + & + & \\
\hline 34 & Metastasis case 11 & 87 & + & neg & neg & \\
\hline 35 & Unique metastasis & 44 & + & neg & neg & \\
\hline 36 & Unique recurrence & 55 & + & + & neg & Extremity \\
\hline 37 & Unique metastasis & 74 & neg & neg & neg & \\
\hline 38 & Unique metastasis & 85 & + & neg & neg & \\
\hline 39 & Unique primary & 79 & + & neg & + & Trunk \\
\hline 40 & Metastasis case 38 & 85 & + & + & + & \\
\hline 41 & Metastasis case 38 & 85 & + & neg & neg & \\
\hline 42 & Unique primary & 80 & neg & neg & neg & \\
\hline 43 & Metastasis case 26 & 72 & + & neg & + & \\
\hline
\end{tabular}

Table 2 Primer sets

\begin{tabular}{|c|c|c|c|c|}
\hline Primer name & Forward sequence $^{\mathrm{a}}$ & Reverse sequence ${ }^{\mathrm{a}}$ & Amplicon size (bp) & Reference \\
\hline MCVPS1 & TCAGCGTCCCAGGCTTCAGA & TGGTGGTCTCСTCTCTGCTACTG & 109 & This study \\
\hline LT1 & TACAAGCACTCCACCAAAGC & TCCAATTACAGCTGGCCTCT & 440 & 10 \\
\hline LT3 & TTGTCTCGCCAGCATTGTAG & ATATAGGGGCCTCGTCAACC & 308 & 10 \\
\hline$\beta$-globin & ACACAACTGTGTTCACTAGC & CAACTTCATCCACGTTCACC & 110 & 16 \\
\hline \multirow[t]{5}{*}{ Size ladder } & GCCCGACATTCTGCAAGTCC & GGTGTTGCCGGGAAGGGTT & 100 & 14 \\
\hline & TGTTGACTCGATCCACCCCA & TGAGCTGCAAGTTTGGCTGAA & 200 & \\
\hline & TGCGATGTGGTCATCATGGTG & CGTGTCATTGTCGTCTGAGGC & 300 & \\
\hline & CCGCAGCAAGCAACGAACC & GCTTTCCTCTGGCGGCTCC & 400 & \\
\hline & GGAGCAGCATTCCATCCAGC & CATCCATGGGCCGGACATAA & 600 & \\
\hline
\end{tabular}

\footnotetext{
${ }^{\mathrm{a}}$ All primer sequences are given in the $5^{\prime}-3^{\prime}$ orientation.
}

well-known consequence of formalin fixation, ${ }^{17}$ it is not surprising that the MCVPS1 primer set (which produces a $109 \mathrm{bp}$ amplicon) detected Merkel cell polyomavirus in more cases of Merkel cell carcinoma than the LT1 and LT3 primer sets (which produce much larger amplicons) (Table 2).
To demonstrate the specificity of the MCVPS1 primer set, we isolated genomic DNA from 40 peripheral blood specimens previously collected for routine genetic screening. Although it is known that Merkel cell polyomavirus is not present in blood (personal communications) it serves as a 


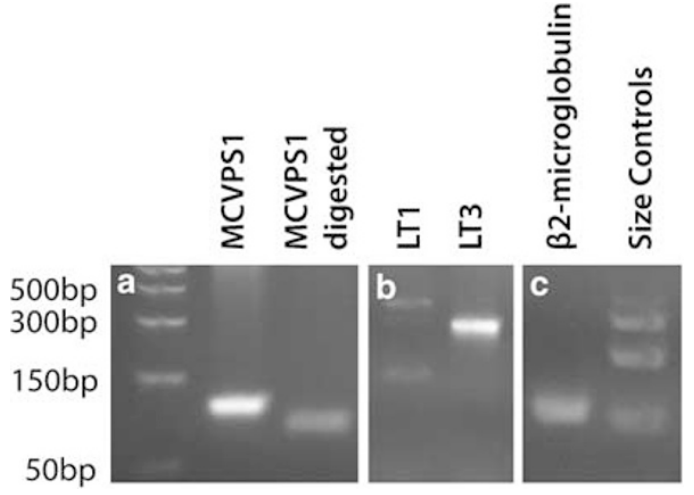

Figure 2 Representative results of the primers used to detect Merkel cell polyomavirus. (a) The $109 \mathrm{bp}$ MCVPS1 PCR product before (left) and after (right) BamH1 digestion that produces an $83 \mathrm{bp}$ product. (b) The LT1 product $(440 \mathrm{bp}$ ) and LT3 product (308 bp); a second nonspecific $100 \mathrm{bp}$ band was frequently seen in LT1 amplifications. (c) $\beta$-globin (110bp) and size amplification controls $(100,200$, and $300 \mathrm{bp}$ bands) run in parallel.

control for nonspecific amplification of genomic DNA with our primer set. None of the 40 samples showed evidence of Merkel cell polyomavirus by the MCVPS1 primer set. In addition, all cases of Merkel cell carcinoma in which Merkel cell polyomavirus was detected by the LT1 and LT3 primers were also positive by the MCVPS1 primer set (Table 1), as expected based on the smaller MCVPS1 amplicon size (as discussed above).

Eleven patients underwent two or more separate excisions. Of these, Merkel cell polyomavirus was detected in both the original and follow-up samples in eight patients. In two patients (cases 18 and 19 and cases 10 and 20), Merkel cell polyomavirus was detected in the primary tumor but not in subsequently excised metastases. In one patient (cases 7 and 15), Merkel cell polyomavirus was detected in a metastatic lesion but not the initial tumor.

\section{Discussion}

Our demonstration that Merkel cell polyomavirus is present in $76 \%$ (22 of 29) of unique cases of Merkel cell carcinoma from formalin-fixed, paraffinembedded tissue makes several important points. First, together with the detection of Merkel cell polyomavirus genomic sequences in paired primary tumors and metastases, it provides support for a link between Merkel cell polyomavirus and the oncogenesis of Merkel cell carcinoma. Second, on a more practical level, our development of a primer set optimized for formalin-fixed, paraffin-embedded tissue, which minimizes the effects of DNA degradation on test sensitivity and produces a product that is easily verified by restriction fragment length analysis, provides a methodology for informative analysis of Merkel cell carcinoma cases encountered in routine surgical pathology practice.

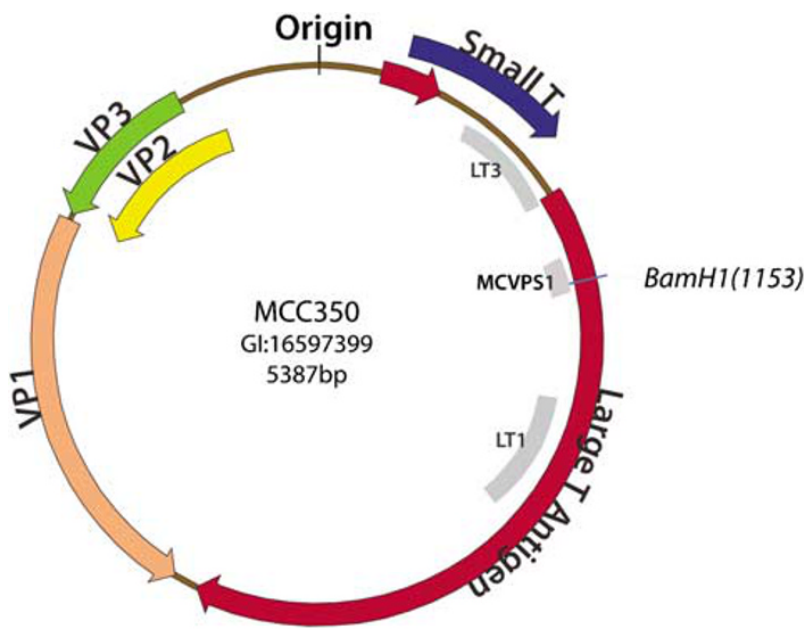

Figure 3 Vector map of the $5387 \mathrm{bp}$ Merkel cell polyomavirus genome. The location of the three primer sets used to detect the virus is shown. All three primer sets are within the 'early gene expression region' (196-3080nt) that contains both large and small $\mathrm{T}$ antigens.

Previous studies have also identified Merkel cell polyomavirus in a high percentage of cases of Merkel cell carcinoma. In the original study that identified and implicated Merkel cell polyomavirus in the oncogenesis of Merkel cell carcinoma, the virus was detected in 8 of 10 cases ( $80 \%)$. However, fresh frozen tissue was the substrate for analysis, and the virus could only be detected in one of the cases by Southern blot analysis of the PCR products. ${ }^{10}$ In a more recent study, Merkel cell polyomavirus was detected in 30 of 39 cases $(77 \%)$ of Merkel cell carcinoma from formalin-fixed paraffinembedded tissue using the LT3 primer set; in comparison, we detected the virus in only 17 of 41 cases $(41 \%)$ using the LT3 primer set, but found a similar percentage of positive cases $(78 \%)$ with our MCVPS1 primer set. The explanation for these differences in test sensitivity using the same primer sets in different laboratories is uncertain, but may be attributable to the age of the tissue blocks, storage conditions, or differences in viral copy number in individual cases. In any event, our testing of $2 \mathrm{~mm}$ tumor cores punched from highly cellular areas of viable tumor using sterile, single-use punches not only increases test sensitivity by enriching the neoplastic cell population but also decreases the risk of cross-contamination.

The fact that three different laboratories have detected Merkel cell polyomavirus genomic sequences in tumor DNA in $75-80 \%$ of Merkel cell carcinoma specimens, ${ }^{10,11}$ together with the demonstration that viral DNA is clonally distributed in Merkel cell carcinoma ${ }^{10,11}$ and is present in primary tumors as well as paired metastases, ${ }^{10}$ provides evidence that Merkel cell polyomavirus has a function in Merkel cell carcinoma oncogenesis. Although a formal etiological link has not been established, in the single Merkel cell carcinoma to date in which the genomic site of integration of 
Merkel cell polyomavirus has been evaluated, the virus is apparently inserted within the human receptor tyrosine phosphatase gene at $3 p 14.2$, a finding that suggests that oncogenesis may occur via insertional mutagenesis as well as T-antigen expression. ${ }^{10}$ As polyomavirus integration is not site specific in the host genome and occurs in the absence of specific viral crossover sites or insertion sequences, ${ }^{18}$ detailed genetic study of the viral integration site in a number of different tumors will likely be required to begin to understand the function of the various host and viral regions involved in Merkel cell carcinoma tumorigenesis.

Although molecular genetic analysis of additional cases is needed to understand the function of Merkel cell polyomavirus in the development of Merkel cell carcinoma, there are a number of questions about the virus that remain unanswered at an epidemiological level. For example, the route of infection is unknown, as is the prevalence of disease. Until the natural history of Merkel cell polyomavirus infection is more well defined, it is impossible to evaluate whether there is a need for screening at-risk populations for the presence of the virus. Such questions are likely to have increasing relevance given the increasing number of immunocompromised individuals within the general population, whether due to HIV infection, organ transplantation, or as a side effect of therapy for autoimmune or neoplastic diseases.

Finally, it will be interesting to establish whether Merkel cell polyomavirus is associated with other neoplasms that occur in immunocompromised populations, or with tumors that do not share similar epidemiological associations but have extremely similar morphological features (eg, highgrade neuroendocrine carcinoma). Our development of a primer set that permits detection of Merkel cell polyomavirus in formalin-fixed, paraffin-embedded tissue with a sensitivity similar to that obtained when fresh tissue is used for analysis broadens the range of specimens that can be studied to begin to address these questions.

\section{Acknowledgements}

We thank Xiaopei Zhu for the expert technical assistance. The secretarial assistance of Elease Barnes is much appreciated. We also thank Walter Clermont for his assistance with digital imaging.

\section{Disclosure/conflict of interest}

The authors have no financial ties to any of the companies mentioned in this paper.

\section{References}

1 Toker C. Trabecular carcinoma of the skin. Arch Dermatol 1972;105:107-110.

2 Tang CK, Toker C. Trabecular carcinoma of the skin: an ultrastructural study. Cancer 1978;42:2311-2321.

3 Plaza JA, Suster S. The Toker tumor: spectrum of morphologic features in primary neuroendocrine carcinomas of the skin (Merkel cell carcinoma). Ann Diagn Pathol 2006;10:376-385.

4 Sibley RK, Dahl D. Primary neuroendocrine (Merkel cell?) carcinoma of the skin. II. An immunocytochemical study of 21 cases. Am J Surg Pathol 1985;9:109-116.

5 LeBoit PE, Burg G, Weeden D, Sarasin A. Pathology and Genetics of Skin Tumors. World Health Organization, IARC Press: Lyon, France, 2006.

6 Hodgson NC. Merkel cell carcinoma: changing incidence trends. J Surg Oncol 2005;89:1-4.

7 Penn I, First MR. Merkel's cell carcinoma in organ recipients: report of 41 cases. Transplantation 1999;68:1717-1721.

8 Rubel JR, Milford EL, Abdi R. Cutaneous neoplasms in renal transplant recipients. Eur J Dermatol 2002 ;12:532-535.

9 Engels EA, Frisch M, Goedert JJ, et al. Merkel cell carcinoma and HIV infection. Lancet 2002;359: 497-498.

10 Feng H, Shuda M, Chang Y, et al. Clonal integration of a polyomavirus in human Merkel cell carcinoma. Science 2008;319:1096-1100.

11 Kassem A, Schopflin A, Diaz C, et al. Frequent detection of Merkel cell polyomavirus in human Merkel cell carcinomas and identification of a unique deletion in the VP1 gene. Cancer Res 2008;68: 5009-5013.

12 Eddy BE, Borman GS, Grubbs GE, et al. Identification of the oncogenic substance in rhesus monkey kidney cell culture as simian virus 40. Virology 1962;17:65-75.

13 zur Hausen H. Novel human polyomaviruses-reemergence of a well known virus family as possible human carcinogens. Int J Cancer 2008;123:247-250.

14 van Dongen JJ, Langerak AW, Bruggemann M, et al. Design and standardization of PCR primers and protocols for detection of clonal immunoglobulin and T-cell receptor gene recombinations in suspect lymphoproliferations: report of the BIOMED-2 Concerted Action BMH4-CT98-3936. Leukemia 2003;17: 2257-2317.

15 Rozen S, Skaletsky H. Primer3 on the WWW for general users and for biologist programmers. Methods Mol Biol 2000;132:365-386.

16 Ferrari A, Casanova M, Bisogno G, et al. Clear cell sarcoma of tendons and aponeuroses in pediatric patients: a report from the Italian and German Soft Tissue Sarcoma Cooperative Group. Cancer 2002;94:3269-3276.

17 Inadome Y, Noguchi M. Selection of higher molecular weight genomic DNA for molecular diagnosis from formalin-fixed material. Diagn Mol Pathol 2003;12:231-236.

18 Wallenburg JC, Nepveu A, Chartrand P. Random and nonrandom integration of a polyomavirus DNA molecule containing highly repetitive cellular sequences. J Virol 1984;50:678-683. 